\title{
ATLAS Plans for the High-Luminosity LHC
}

\author{
Wolfgang Walkowiak* on behalf of the ATLAS Collaboration \\ University of Siegen, 57068 Siegen, Germany \\ E-mail: walkowiak@hep.physik.uni-siegen.de
}

\begin{abstract}
Despite the excellent performance of the Large Hadron Collider (LHC) at CERN an upgrade to a High-Luminosity LHC (HL-LHC) with a peak instantaneous luminosity of up to $7.5 \times 10^{34} \mathrm{~cm}^{-2} \mathrm{~s}^{-1}$ will be required after collecting a total dataset of approximately $300 \mathrm{fb}^{-1}$ by the end of Run 3 (in 2023). The upgrade will substantially increase the statistics available to the experiments for addressing the remaining open puzzles of particle physics. The HL-LHC is expected to start operating in 2026 and to deliver up to $4000 \mathrm{fb}^{-1}$ within twelve years. The corresponding upgrades of the ATLAS detector and the ATLAS beauty physics program at the HL-LHC are being discussed. As examples, preliminary results on the expected sensitivities for the search for CP-violation in the decay channel $B_{s}^{0} \rightarrow J / \psi \phi$ using the parameters $\Delta \Gamma_{s}$ and $\phi_{s}$ as well as projections for the branching fractions of the rare decays $B_{s}^{0} \rightarrow \mu^{+} \mu^{-}$and $B^{0} \rightarrow \mu^{+} \mu^{-}$ are provided.
\end{abstract}

The International Conference on B-Physics at Frontier Machines - BEAUTY2018

6-11 May, 2018

La Biodola, Elba Island, Italy

${ }^{*}$ Speaker. 


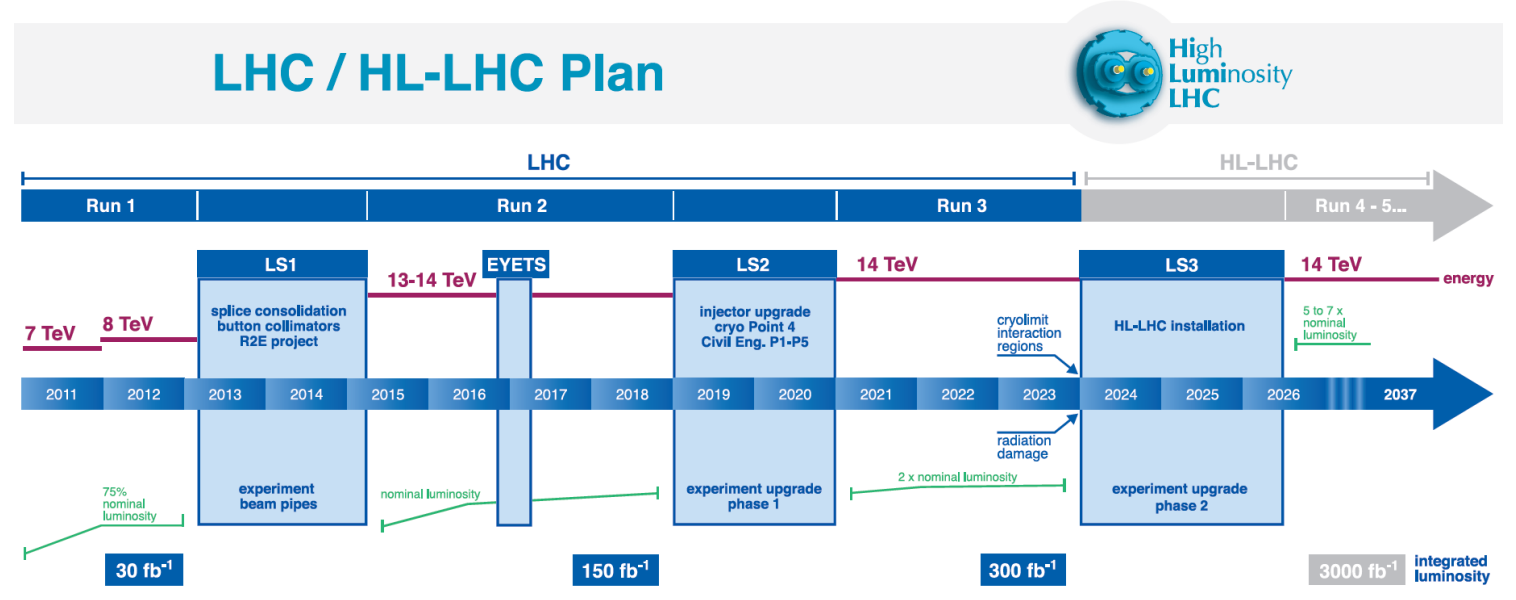

Figure 1: Upgrade program for the LHC accelerator complex [2]. The status of the past seven years and the plans for the next two decades are shown.

\section{Introduction}

The Standard Model (SM) of particle physics describes a broad range of observations well while many open questions such as dark matter, the matter-antimatter asymmetry, neutrino masses or possible flavor anomalies are not easily described by the same framework. In order to extend the successful physics program at the LHC [1] to high precision studies and searches for new phenomena substantially more data needs to be collected. Examples include the detailed investigation of the production and decay of the SM Higgs boson or of CP-violating parameters in other sectors like the $B_{s}^{0} \rightarrow J / \psi \phi$ decay as well as searches for Supersymmetry (SUSY) and other new physics beyond the Standard Model, like in the very rare decay $B^{0} \rightarrow \mu^{+} \mu^{-}$. Therefore an extensive upgrade program for the LHC [2] and the experiments has been implemented (Fig. 1). The high-luminosity LHC (HL-LHC) aims to provide 3000 to $4000 \mathrm{fb}^{-1}$ within approximately 12 years, which is more than 10 times the integrated luminosity of the LHC Runs 1-3 combined. The HL-LHC baseline parameters with a peak luminosity $\mathscr{L} \approx 5 \ldots 7.5 \times 10^{34} \mathrm{~cm}^{-2} \mathrm{~s}^{-1}$ and an average $\langle\mu\rangle \approx 140 \ldots 200$ $p p$ interactions every $25 \mathrm{~ns}$ considerably exceed the nominal LHC design parameters and present a challenge to the existing experiments.

\section{ATLAS Upgrade Program}

In order to profit from the increased luminosity at the HL-LHC and to cope with the harsher operational conditions a major upgrade [3, 4] of the current ATLAS detector [5] is planned.

During the phase-2 upgrade the current Inner Detector (ID) will be replaced by an all-silicon Inner Tracker (ITk), see Fig. 2 left. The new ITk pixel detector [6] consists of $\sim 13 \mathrm{~m}^{2}$ silicon pixel modules with $\sim 580$ million channels ( $\sim 92$ million now), organized into 5 barrel and 5 endcap layers (with sub-rings) providing increased coverage up to $|\eta|=4.0(|\eta|=2.5$ now). The innermost barrel layer is located at $R=36 \mathrm{~mm}$ from the beam axis and the pixel modules at the ends of the barrel layers are inclined to allow for lower incident angles of particles. The new ITk 

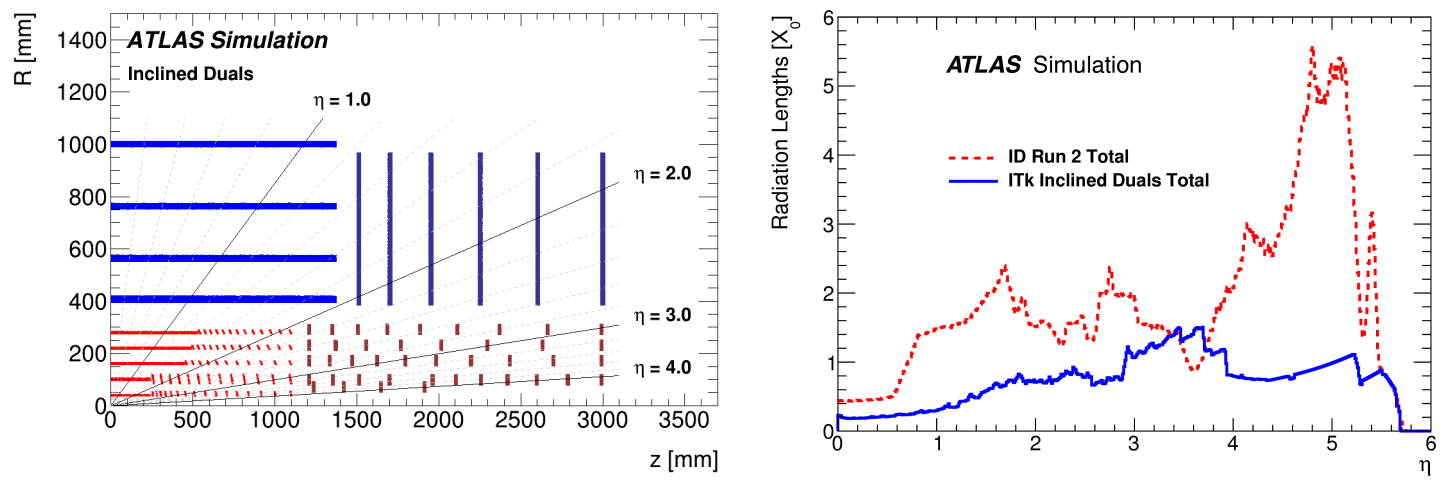

Figure 2: Left: A schematic layout of the ITk Inclined Duals layout for the HL-LHC phase as presented in [6]. Right: A comparison of the material budget in radiation length $X_{0}$ of the ITk and the current ATLAS Inner Detector [6].

strip detector [7] comprises $\sim 160 \mathrm{~m}^{2}$ of silicon strip detector modules in 4 cylindrical barrel and 6 end-cap rings, a total of $\sim 50$ million channels ( $\sim 6$ million now). The strip system will cover $|\eta| \leq 2.5$ with an expected strip occupancy of less than $1 \%$. Compared to the current ATLAS Inner Detector, the ITk features a reduced material budget (Fig. 2 right) resulting in an improved tracking efficiency and better invariant mass resolution of reconstructed particles.

For the upgrade of the ATLAS muon system, already during the phase-1 upgrade New Small Wheels (NSW) [8] are inserted between the end-cap calorimeters and the end-cap toroid magnets (Fig. 3 left). The NSW provide high-efficiency hits for a fast L1 trigger by Small strip Thin Gap Chambers (sTGC) and contribute to the precision measurement of muon tracks with MicroMegas (MM). Their coverage in $1.3<|\eta|<2.7$ will reduce the rate of fake tracks in a high radiation background region. During the phase-2 upgrade [9] new inner barrel (BI) RPC chambers will be inserted as the innermost muon layer (Fig. 3 left), increasing the L0 trigger acceptance $\times$ efficiency for reconstructed combined muons within $|\eta|<1.05$ from $78 \%$ to $96 \%$. Even in the "worst case" scenario with the other RPC stations running at reduced high voltage (due to aging effects) the trigger with the BI RPCs is expected to reach an acceptance $\times$ efficiency of $92 \%$ (instead of 57\% to $75 \%$ without), see Fig. 3 right.

The phase 2 baseline design of the ATLAS trigger and data acquisition (DAQ) system [10] consists of a L0 hardware trigger based on calorimeter and muon system information with an output rate of $1 \mathrm{MHz}$ at $10 \mu \mathrm{s}$ latency, an Event Filter combining a Hardware Track Trigger (HTT) with software-based reconstruction on a large processor farm providing output at $10 \mathrm{kHz}$, as well as a new DAQ system based on the Front ENd LInk eXchange (FELIX) architecture common to all detector systems and a new storage handler. At L0, precision information from the muon system is available and a global event processor constructs refined physics objects $\left(e^{ \pm}, \gamma, \tau\right.$, jet and $\left.E_{T}^{\text {miss }}\right)$. As an option which may also be exercised later a dual L0/L1 trigger with hardware tracking (L1track) for additional pileup suppression and with an increased L0/L1 output rate of $4 \mathrm{MHz}$ at $10 \mu$ s latency is being considered.

All detector systems will upgrade their readout electronics to match the new DAQ system's increased readout speeds. 

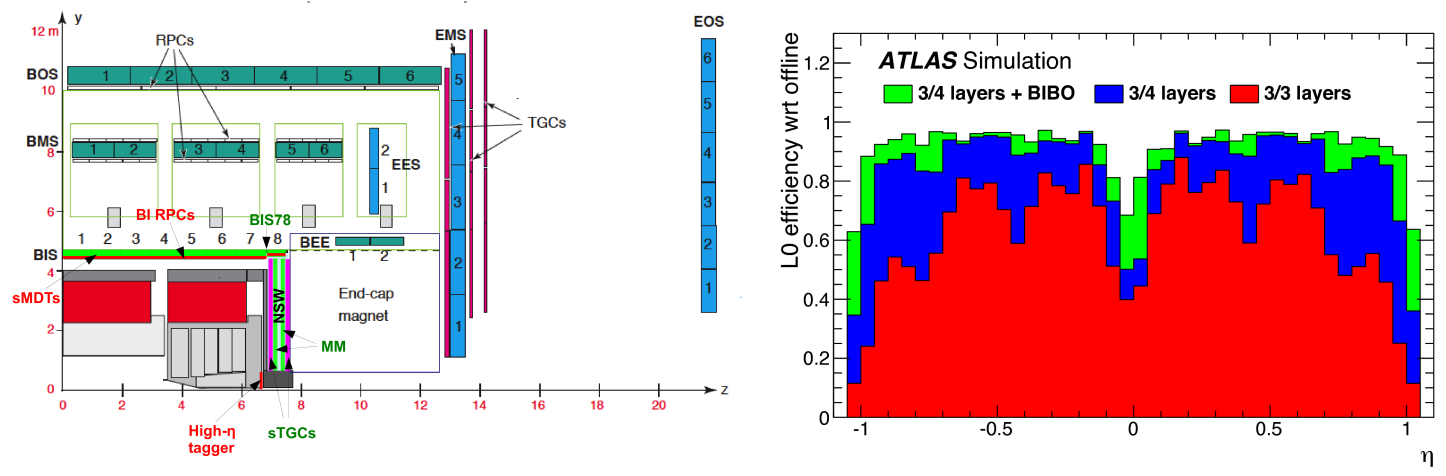

Figure 3: Left: 1.3: An $R-Z$ view of the Phase-II ATLAS muon spectrometer layout showing a small sector [9]. The drawings show the new detectors to be added in the Phase-II upgrade, including the addition of the high- $\eta$ tagger (red text: BI RPC, sMDT, EIL4 TGC, high- $\eta$ tagger), those to be installed during Long Shutdown 2 (green text: Micromegas and sTGC in the New Small Wheel and BIS78 RPC and sMDT), and those that will remain unchanged from the Run 1 layout (black text).

Right: Acceptance $\times$ efficiency of the RPC trigger with respect to reconstructed muons as a function of $\eta$ for the "worst case" hit efficiency scenario in which the RPC HV is reduced to maintain the chamber currents within a safe limit with a safety factor of two. The red histograms show the efficiency of the existing " $3 / 3$ chambers" trigger, the blue histograms the " $3 / 4$ chambers" trigger, and the green histograms the " $3 / 4$ chambers + BI-BO" trigger. The values are evaluated by the Monte-Carlo (MC) samples of muons with a fixed $p_{T}$ of $25 \mathrm{GeV}$ and no pile-up involved [10].

\section{ATLAS B-Physics Prospects at the High-Luminosity LHC}

The ATLAS B-physics program at the HL-LHC encompasses precision measurements of $B_{s}^{0} \rightarrow$ $J / \psi \phi$ or $\Lambda_{b}^{0} \rightarrow J / \psi \Lambda^{0}$ and rare processes like $B_{(s)}^{0} \rightarrow \mu^{+} \mu^{-}$and $b \rightarrow s \mu^{+} \mu^{-}$to search for beyondSM effects, the search for lepton flavor violation and test of lepton flavor universality e.g. in $\tau \rightarrow 3 \mu, B_{s}^{0} \rightarrow e \mu$ or $B^{0} \rightarrow K^{* 0} e^{+} e^{-} / B^{0} \rightarrow K^{* 0} \mu^{+} \mu^{-}$, as well as heavy flavor production to test QCD predictions, to study double parton scattering and to search for new/exotics states or new decay modes.

The angular distributions in the decay $B_{s}^{0} \rightarrow J / \psi \phi$ with $J / \psi \rightarrow \mu^{+} \mu^{-}$and $\phi \rightarrow K^{+} K^{-}$are sensitive to the CP-violating phase $\phi_{s}$. The ATLAS Run 1 result [11] of $\phi_{s}=-0.090 \pm 0.078$ (stat) \pm 0.041 (syst) rad agrees well with the SM and measurements by other experiments [12] while there is still room for New Physics in CP-violation. The sensitivity to $\phi_{s}$ as well as to the $B_{s}^{0}$ decay width difference $\Delta \Gamma_{s}$ is improved considerably by the detector upgrades. As shown in Fig. 4 the addition of the Insertable B-Layer (IBL) [13] in Run 2 improved the proper decay time resolution $\sigma_{t}$ by $\sim 30 \%$. A dedicated study [7] with dedicated MC signal samples with $\langle\mu\rangle=200$ pile-up events and $\mathscr{L}=7 \times 10^{34} \mathrm{~cm}^{-2} \mathrm{~s}^{-1}$ for an ITk layout with $50 \times 50 \mu \mathrm{m}^{2}$ pixels and the innermost pixel layers at $R=39 \mathrm{~mm}$ and $80 \mathrm{~mm}$ from the beamline shows further improvement, especially at higher $p_{T}\left(B_{s}^{0}\right)$. Fig. 4 right demonstrates the very good stability of $\sigma_{t}$ as a function of the number of reconstructed vertices. The additional gain expected from the use of analogue instead of digital pixel clustering is not yet included. An earlier study [14] for ECFA 2013 with simulated signal 

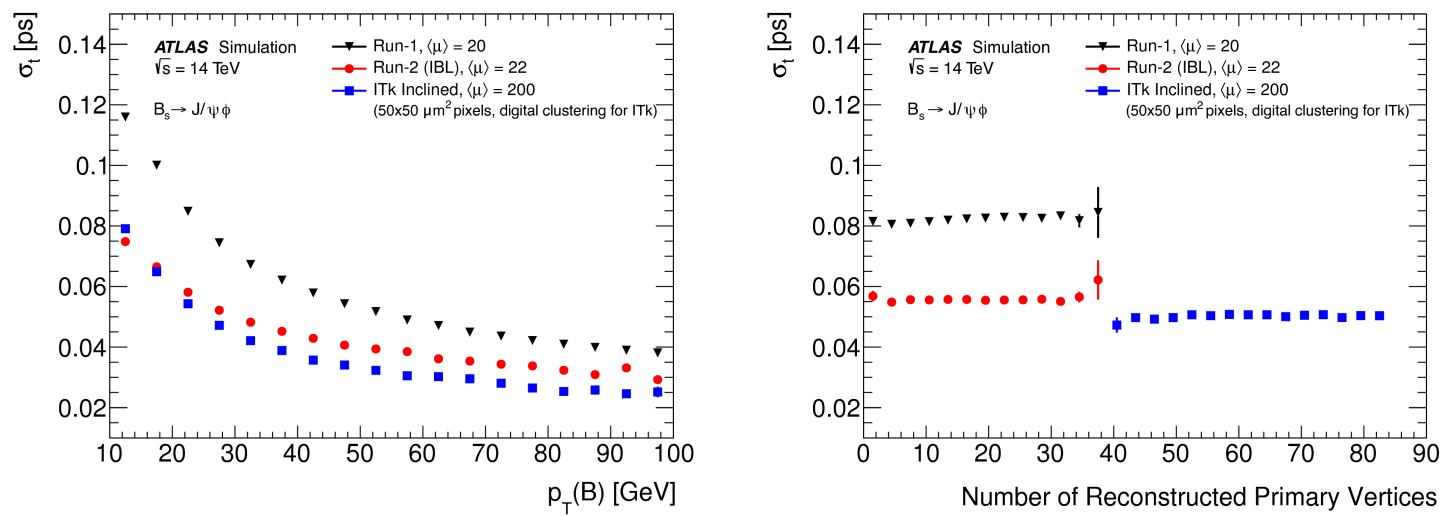

Figure 4: The average $B_{s}^{0}$ proper decay time resolution st as a function of the transverse momentum $p_{T}$ of the $B_{s}^{0}$-meson (left) and stability of the average $B_{s}^{0}$ proper decay time resolution against the number of reconstructed primary vertices (right) of simulated $B_{s}^{0} \rightarrow J / \psi \phi$ decays, shown for ITk (blue), the Run 1 detector (black) and the Run 2 detector including the IBL (red), for their respective pile-up conditions [6].

MC events and fits to pseudo-experiments based on the Run 1 analysis strategy demonstrates the statistical reach for an integrated luminosity of $3000 \mathrm{fb}^{-1}$ resulting in $\sigma\left(\phi_{s}\right) \approx 0.022 \mathrm{rad}$. A later study [15] indicates a further potential improvement of a factor 3 by the use of topological muon triggers which will allow for lower $p_{T}(\mu)$ trigger thresholds. The systematic uncertainties $\left(B_{s}^{0}\right.$ flavor tagging calibration, likelihood fit model description, fit to $B^{0} \rightarrow J / \psi K^{* 0}$ component, trigger efficiency and Inner Detector alignment) are expected to improve with increased statistics as well.

The branching fraction measurement of the very rare decays $B_{s}^{0} \rightarrow \mu^{+} \mu^{-}$and $B^{0} \rightarrow \mu^{+} \mu^{-}$which are sensitive to New Physics in the decays via loop diagrams will benefit from the increased statistics and the improved invariant mass resolution at the HL-LHC. The ATLAS Run 1 result [16] is compatible with the SM at $\sim 2 \sigma$ level and the $\mathscr{B}\left(B_{(s)}^{0} \rightarrow \mu^{+} \mu^{-}\right)$values are lower than the CMS-LHCb combined result (Fig. 5 left). A study [6] of the $B_{s}^{0}$ mass resolution with simulated $B_{s}^{0} \rightarrow \mu^{+} \mu^{-}$MC events for Run 2 conditions as in 2015 and for the HL-LHC with $\langle\mu\rangle=200$ pileup events and $\mathscr{L}=7 \times 10^{34} \mathrm{~cm}^{-2} \mathrm{~s}^{-1}$ for an ITk layout with $50 \times 50 \mu \mathrm{m}^{2}$ pixels covering $|\eta|<4.0$ and applying an analysis strategy similar to the Run 1 analysis shows a considerable improvment (Fig. 5 right). The separation of the $B_{s}^{0}$ and $B_{d}^{0}$ mass peaks increases by a factor of 1.65 (1.5) to $2.3 \sigma(1.3 \sigma)$ in the barrel (end-cap) region compared to Run 1 [15].

The projection of the ATLAS detector performance for measuring $\mathscr{B}\left(B_{(s)}^{0} \rightarrow \mu^{+} \mu^{-}\right)$with the expected datasets during the full LHC Run $2\left(130 \mathrm{fb}^{-1}\right)$ and at the HL-LHC $\left(3000 \mathrm{fb}^{-1}\right)$ [19] using pseudo-MC experiments is based on the likelihood of the Run 1 analysis. The signal statistics estimate for the Run 2 scenario applies scaling factors for the integrated luminosity, the cross-section increase due to the higher center-of-mass energy of 13 or $14 \mathrm{TeV}$ and the muon pair selection with topological triggers with $\left(p_{T}\left(\mu_{1,2}\right)>6 \mathrm{GeV}\right)$ or $\left(p_{T}\left(\mu_{1}\right)>6 \mathrm{GeV}, p_{T}\left(\mu_{1}\right)>4 \mathrm{GeV}\right)$ thresholds resulting in 7 times the number of signal events in Run 1 . The contours of the 2-dimensional Neyman construction (Fig. $6(a)$ ) include the external systematic uncertainties on the $b$-quark fragmentation fractions $f_{s} / f_{d}$ and $\mathscr{B}\left(B^{ \pm} \rightarrow J / \psi K^{ \pm}\right)$which were kept the same as in the Run 1 analysis as well as internal ones like the fit shapes and efficiencies which were scaled according to the increase in 

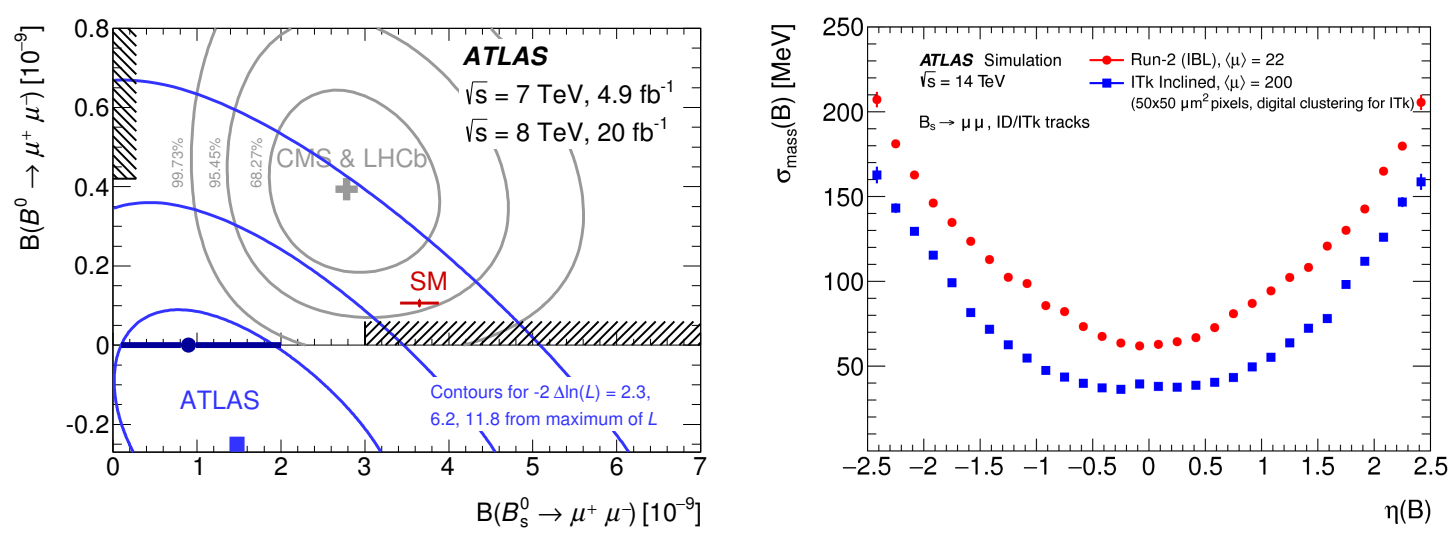

Figure 5: Left: ATLAS Run 1 contours in the plane $\mathscr{B}\left(B_{s}^{0} \rightarrow \mu^{+} \mu^{-}\right), \mathscr{B}\left(B^{0} \rightarrow \mu^{+} \mu^{-}\right)$for intervals of $-2 \Delta \ln (L)$ equal to $2.3,6.2$ and 11.8 relative to the absolute maximum of the likelihood, without imposing the constraint of non-negative branching fractions. For details see [16]. Also shown are the likelihood maximum and the corresponding contours for the combined result of the CMS and LHCb experiments [17], and the SM prediction [18].

Right: Comparison of the Run 2 and HL-LHC $B_{s}^{0} \rightarrow \mu^{+} \mu^{-}$mass resolution as a function of the $B_{s}^{0}$-meson pseudorapidity using only the ID/ITk track parameter measurement [6].

statistics. For the HL-LHC case three potential trigger scenarios are considered: two muons with $p_{T}>10 \mathrm{GeV}$ ("conservative"), one muon with $p_{T}>10 \mathrm{GeV}$ and another with $p_{T}>6 \mathrm{GeV}$ ("intermediate") as well as two muons with $p_{T}>6 \mathrm{GeV}$ ("high yield") providing 15, 60 and 70 times the Run 1 statistics, respectively. The profile likelihood contours of pseudo-experiments based again on the likelihood of the Run 1 analysis demonstrate the increased sensitivity of the ATLAS detector for $\mathscr{B}\left(B_{s}^{0} \rightarrow \mu^{+} \mu^{-}\right)$and $\mathscr{B}\left(B^{0} \rightarrow \mu^{+} \mu^{-}\right)$at the HL-LHC (Fig. $\left.6(b)-(d)\right)$. The uncertainty on the $f_{s} / f_{d}$ value, conservatively taken as $8.3 \%$ from the ATLAS measurement [20], dominates the systematic uncertainty contributions on $\mathscr{B}\left(B_{s}^{0} \rightarrow \mu^{+} \mu^{-}\right)$.

\section{Summary}

To profit from the HL-LHC with $\sim 5$ times the LHC's peak instantaneous luminosity many components of the ATLAS detector will be upgraded. The ATLAS B physics program will continue during LHC Run 3 and at the HL-LHC with a similar focus as during LHC Run 1 and Run 2. It will exploit the detector upgrades, especially the new Inner Tracker and in the muon system, which provide an improved secondary vertex reconstruction and a better invariant mass resolution. The development of topological L0/L1 triggers to keep low lepton $p_{T}$ thresholds will be important. The increased data statistics will be used for precision measurements and searches, e.g. for CP-violation in the $B_{s}^{0} \rightarrow J / \psi \phi$ decay or for rare decays like $B_{(s)}^{0} \rightarrow \mu^{+} \mu^{-}$.

\section{Acknowledgments}

This work was partially supported by grants of the German Federal Ministry of Education and Research (BMBF) and the German Helmholtz Alliance "Physics at the Terascale". 

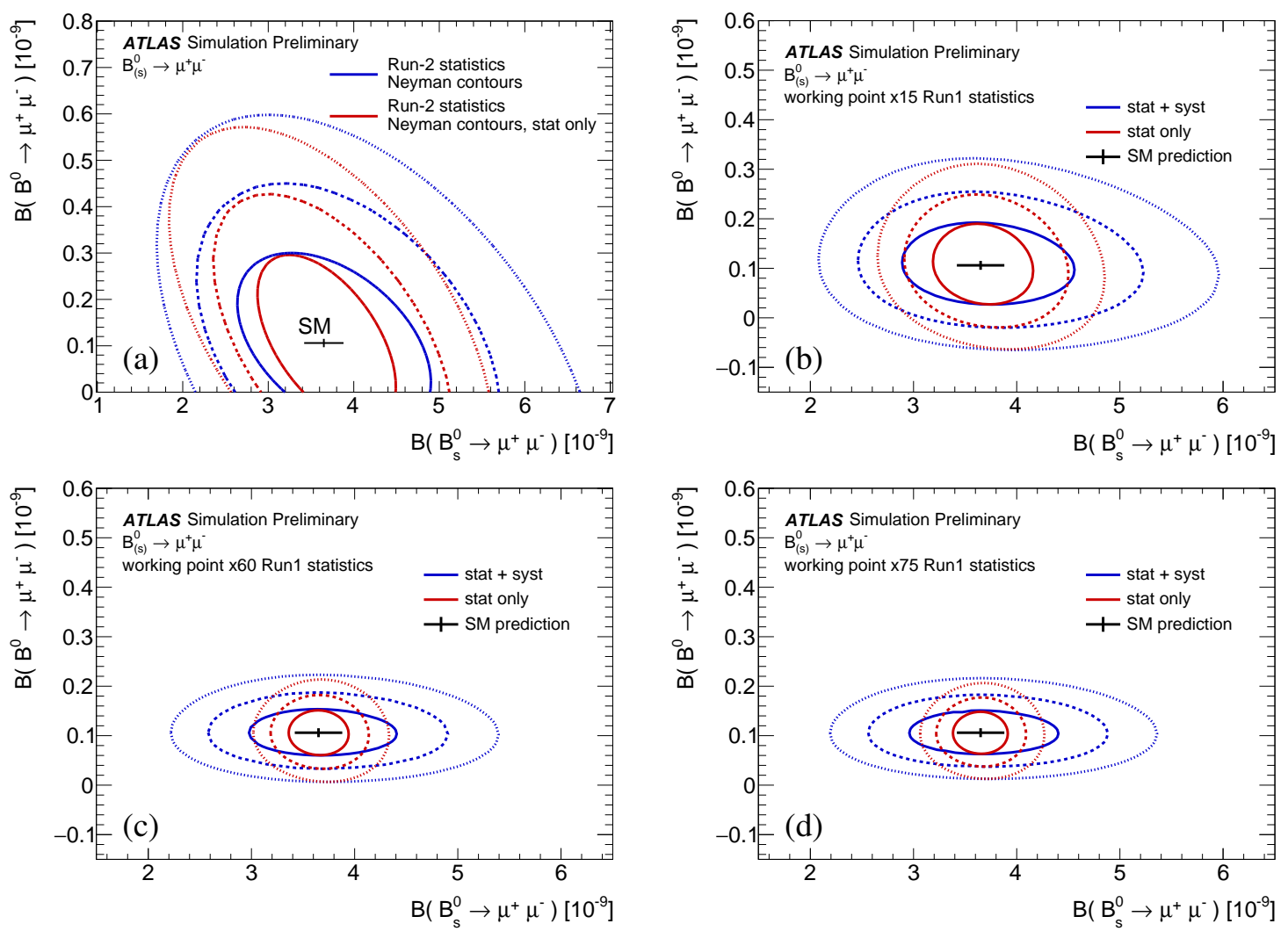

Figure 6: (a): Comparison of $68.3 \%$ (solid), $95.5 \%$ (dashed) and $99.7 \%$ (dotted) confidence level contours obtained exploiting the 2D Neyman belt construction for the Run 2 case [19]. Red contours are statistical only; blue contours include systematics uncertainties from the ATLAS Run 1 analysis [16] extrapolated to Run 2 statistics. The black points show the SM theoretical prediction and its uncertainty [18].

(b) - $(d)$ : Comparison of confidence level profiled likelihood ratio contours for $(b)$ the 'conservative', $(c)$ the 'intermediate' and $(d)$ the 'high-yield' HL-LHC extrapolation with $\times 15, \times 60$ and $\times 75$ the Run 1 statistics for the $(10 \mathrm{GeV}, 10 \mathrm{GeV})$, the $(6 \mathrm{GeV}, 10 \mathrm{GeV})$ and the $(6 \mathrm{GeV}, 6 \mathrm{GeV})$ dimuon trigger scenarios, respectively [19].

\section{References}

[1] L. Evans and P. Bryant (editors), LHC Machine, 2008 JINST 3 S08001

[2] G. Apollinari, I. Béjar Alonso, O. Brüning, P. Fessia, M. Lamont, L. Rossi, L. Tavian (editors), High-Luminosity Large Hadron Collider (HL-LHC), Technical Design Report V. 0.1, CERN Yellow Reports Vol. 4/2017, CERN-2017-007-M (CERN, Geneva, 2017)

[https://cds.cern.ch/record/2284929]

[3] ATLAS Collaboration, Letter of Intent for the Phase-II Upgrade for the ATLAS Experiment, Tech. Rep. CERN-LHCC-2012-022, LHCC-I-023, CERN, 2012

[https://cds.cern.ch/record/1502664]

[4] ATLAS Collaboration, ATLAS Phase-II Upgrade Scoping Document, Tech. Rep. CERN-LHCC-2015-020, LHCC-G-166, CERN, 2015

[https://cds.cern.ch/record/2055248] 
[5] ATLAS Collaboration, The ATLAS Experiment at the CERN Large Hadron Collider, 2008 JINST 3 S08003

[6] ATLAS Collaboration, Technical Design Report for the ATLAS Inner Tracker Pixel Detector, CERN-LHCC-2017-021, ATLAS-TDR-030 [https : / / cds.cern.ch/record/2285585]

[7] ATLAS Collaboration, Technical Design Report for the ATLAS Inner Tracker Strip Detector, CERN-LHCC-2017-005, ATLAS-TDR-025 [https : / / cds . cern. ch/record/2257755]

[8] ATLAS Collaboration, ATLAS New Small Wheel Technical Design Report, CERN-LHCC-2013-006, ATLAS-TDR-20-2013 [https: / / cds . cern.ch/record/1552862]

[9] ATLAS Collaboration, Technical Design Report for the Phase-II Upgrade of the ATLAS Muon Spectrometer, CERN-LHCC-2017-017, ATLAS-TDR-026

[https://cds.cern.ch/record/2285580]

[10] ATLAS Collaboration, Technical Design Report for the Phase-II Upgrade of the ATLAS Trigger and Data Acquisition System, CERN-LHCC-2017-020, ATLAS-TDR-029

[https://cds.cern.ch/record/2285584]

[11] ATLAS Collaboration, Measurement of the CP-violating phase $\phi_{s}$ and the $B_{s}^{0}$ meson decay width difference with $B_{s}^{0} \rightarrow J / \psi \phi$ decays in ATLAS, JHEP 08 (2016) 147

[12] Y. Amhis et al., Averages of b-hadron, c-hadron and tau-lepton properties as of summer 2016, Eur. Phys. J. C77 (2017) 895 [arXiv: 1612 . 07233]

[13] ATLAS Collaboration, Production and Integration of the ATLAS Insertable B-Layer, 2018 JINST 13 T05008 [arXiv: 1803.00844 ]

[14] ATLAS Collaboration, ATLAS B-physics studies at increased LHC luminosity, potential for $C P$-violation measurement in the $B_{s}^{0} \rightarrow J / \psi \phi$ decay, ATL-PHYS-PUB-2013-010 [http: / / cdsweb.cern.ch/record/1604429]

[15] ATLAS Collaboration, Expected peformance for an upgraded ATLAS detector at High-Luminosity LHC, ATL-PHYS-PUB-2016-026 [https : / / cds . cern. ch/record/2223839]

[16] ATLAS Collaboration, Study of the rare decays of $B_{s}^{0}$ and $B_{d}^{0}$ into muon pairs from data collected during the LHC Runl with the ATLAS detector, Eur. Phys. J. C (2016) 76:513 [arXiv: 1604.04263$]$

[17] CMS and LHCb Collaborations, Observation of the rare $B_{s}^{0} \rightarrow \mu^{+} \mu^{-}$decay from the combined analysis of CMS and LHCb data, Nature, 522, 2015

[18] C. Bobeth et al, $B_{s, d}^{0} \rightarrow \ell^{+} \ell^{-}$in the Standard Model with Reduced Theoretical Uncertainty, Phys. Rev. Lett. 112 (2014) 101801 [arXiv: 1311.0903 ]

[19] ATLAS Collaboration, Prospects for the $\mathscr{B}\left(B_{(s)}^{0} \rightarrow \mu^{+} \mu^{-}\right)$measurements with the ATLAS detector in Run 2 LHC and HL-LHC data campaigns, ATL-PHYS-PUB-2018-005 [https://cds.cern.ch/record/2317211]

[20] ATLAS Collaboration, Determination of the ratio of b-quark fragmentation fractions $f_{s} / f_{d}$ in $p p$ collisions at $\sqrt{s}=7$ TeV with the ATLAS detector, Phys. Rev. Lett. 115, 262001 (2015) 